\title{
Participación en deporte escolar en la Región de Murcia: centros públicos versus concertados-privados
}

\section{Participation in school sport in the Region of Murcia: public versus semi-private and private centers}

\author{
V. Morales, A. Martínez-Moreno y A. Díaz \\ Facultad Ciencias del Deporte. Universidad de Murcia
}

\begin{abstract}
Resumen: Tomando como fuente de información datos de la Dirección General de Actividad Física y Deportes de la Región de Murcia, en este estudio se delimitaron el número de participaciones, ratio de participación, media de participación por población, es decir, cuantas participaciones por individuo se producen en la población en relación al Deporte en edad Escolar en la Región de Murcia durante los cursos 2005 hasta el 2010. La muestra está formada por 155.009 participaciones masculinas y 94.317 femeninas de colegios públicos y privados-concertados. La participación en los centros privados-concertados fue $24,83 \%$ con 193.628 matriculados frente a $36,72 \%$ en los públicos con 548.100 matriculados. La participación en relación al ratio por individuo también se decanto a favor de los colegios públicos 0,457 frente a los 0,268 de los privados-concertados. Los datos mostraron que es mayor el número de participaciones en términos relativos y absolutos de los centros públicos.

Palabras clave: Deporte Escolar, participación, Educación Física.
\end{abstract}

Abstract: It is considered as a source the data from the General Directorate of the Physical Activity and Sports (Dirección General de Actividad Física y Deportes) in the Region of Murcia. In this study, it is delimited the number of participants, ratio of participation and the average of the participation in relation to the population, that is, how many participations per person are taken place relating to Sport in school-age in the Region of Murcia during the period of the academic years from 2005 to 2010. The sample is formed by 155.009 masculine participants and 94.317 feminine participants from public and state-subsidized private schools. The participation in state-subsidized private schools was $24,83 \%$ (193.628 registered) versus the $36,72 \%$ (548.100 registered) in public schools. The participation in relation to the ratio per person was also biased in favor of the public schools $(0,457$ facing the 0,268 of the state-subsidized private schools). Therefore, data showed that the number of participations in relative and absolute terms is greater in public schools.

Keywords: School sport, Participation, Physical Education.

\section{Introducción}

El marco escolar se sitúa como pilar básico de desarrollo deportivo, siendo los modelos menos tecnológicos, más emocionales, colectivos y universales. Todos tienen cabida, tanto los talentos deportivos como los menos dotados para la práctica motriz. Destacando algunos objetivos presentes en este ámbito: las relaciones sociales, los hábitos de salud, la motivación, la autoestima, el control de la agresividad, el conocimiento de uno mismo, el respeto a los demás, el juego limpio, etc. Esta actividad cumple diversas funciones sociales, a veces de manera contradictoria, presenta múltiples realidades y en todas ellas subyace una forma de entender, presenciar y realizar dicha práctica (Díaz y Martínez-Moreno, 2003).

Las principales señas de identidad de la idea de deporte: situación motriz con cierto grado de intervención intelectual, logro de resultado deportivo, en competición o no. Para ello, se requiere una preparación o entrenamiento más o menos estructurado. Sus criterios de éxito están regulados por un reglamento o, en su defecto por un código ético espontáneo y

\footnotetext{
Dirección para correspondencia [Correspodence address]: A. MartínezMoreno. Facultad Ciencias del Deporte. Universidad de Murcia (España). E-mail: dr.almamo@gmail.com
}

flexible, que generalmente se institucionalizan en el contexto de organizaciones sociales como asociaciones o federaciones. (Devís, 1995; Moreno-Hernández, 1998; Pérez-Brunicardi, 2011)

Dentro de este complejo término, el ámbito más necesitado de atención es el deporte escolar, porque supone la base del desarrollo deportivo de una sociedad. Los grandes cambios en los hábitos deportivos, de salud y de calidad de vida de los ciudadanos, si han de venir, tendrán su base en la escuela y hacer caso omiso a esta realidad es un signo de estancamiento social cuya responsabilidad recae sobre las personas y entidades con competencias en este ámbito. La práctica deportiva constituye uno de los fenómenos sociales de más envergadura, dentro de las sociedades avanzadas contemporáneas (Sánchez, 1996); y si la consolidación de hábitos saludables de práctica física tiene lugar en las etapas tempranas de la vida (Blair, Dunn, García, Kampert, Kohl \& Marcus, 1999), no se alcanzará el mínimo éxito si no se afronta de lleno lo que ocurre con los niños y adolescentes (Piéron, Telama, Almond, y Carreiro da Costa, 1999 y Gutiérrez, 2000).

El deporte en la escuela hace referencia al desarrollado en el entorno de un centro escolar, sometido a su estructura y 
con una incidencia directa del profesorado del centro. Mientras, el deporte en edad escolar es un concepto más amplio que recoge todas aquellas actividades deportivas ofrecidas al niño dentro de un periodo temporal más o menos concreto (Moreno-Hernández, 1998). Son muchos los autores que de una u otra forma han realizado aportaciones para determinar o aclarar el concepto Elías y Dunnig (1992), Blázquez (1995), Devís (1995), Díaz \& Martínez-Moreno (2003), López (2006), Rodríguez (2010), Bustamante et al. (2011) desbordando los límites escolares como espacio físico y apoyándose en el ámbito de los requerimientos sociales y formativos (González et al., 2015)

Si hablamos de los Juegos Escolares, es preceptivo recoger que, aunque en su inicio el modelo que les sustentaba era esencialmente: competitivo, excluyente, por sexos y basado en sistemas eliminatorios de participación, poco a poco van evolucionando, a pesar de que parece que les cuesta más a unos deportes concretos y a unas comunidades autónomas más que a otras. Díaz y Martínez (2003) denuncian que este es un enfoque selectivo, excluyente y apropiado para los más capacitados, reproduce la idea de la segregación de sexos y tiende a minimizar el carácter lúdico y recreativo. Incorpora algunos valores más propios del mundo de la alta competición, de ética y moral más que dudoso.

Alrededor del deporte escolar se genera un engranaje formado por diferentes grupos de personas e instituciones que contribuyen y participan en el desarrollo del proyecto que se diseńa y que se lleva a cabo bajo unas determinadas circunstancias y en unas condiciones específicas que permitirán desarrollarlo más o menos adecuadamente. Los agentes implicados en el deporte en edad escolar para Díaz y MartínezMoreno (2003) son: Administración pública, centro escolar, profesores de Educación Física, técnicos deportivos, entidades deportivas y familias. Influyendo en las intenciones futura de práctica deportiva las metas y objetivos propuestos (Cecchini et al., 2008).

Muchas son las voces que plantean la necesidad de huir de planteamientos estancos de deporte para edades escolares, sin asegurarse de caminar en la misma dirección. Esta es la educación integral de los alumnos. Numerosos autores (Álamo, 2004; García-Aranda, 2010; González, 2004 y Santos y Sicilia, 1998) que plantean fórmulas e ideas para realizar la conexión entre el considerado deporte extraescolar y el deporte incluido dentro del ámbito escolar.

En la Ley del Deporte en la Región de Murcia (2000), la Consejería de Presidencia expone las condiciones que debe tener el deporte escolar. En el Preámbulo de la Ley del Deporte de la Región de Murcia se cita el siguiente texto sobre el deporte y su práctica: El deporte, en sus múltiples y muy varias manifestaciones, se ha convertido en nuestro tiempo en una de las actividades sociales con mayor arraigo y capacidad de movilización y convocatoria. En su Título II. Educación, salud y deporte. Capítulo I Del Deporte Escolar. Artículo 10. Concepto. 1. Se considera como deporte escolar a los efectos de esta ley, aquella actividad deportiva organizada que es practicada por escolares en horario no lectivo. 2. Su práctica será preferentemente polideportiva y no orientada exclusivamente a la competición, de tal manera que se garantice que todos los escolares conozcan la práctica de diversas

El término deporte en edad escolar se encuentra muy próximo semánticamente al de deporte escolar, aunque es más amplio (Abad, Robles y Giménez, 2009; Pérez-Brunicardi, 2011). Por tanto, podríamos decir que el deporte en edad escolar es la práctica deportiva realizada durante el periodo de escolarización obligatoria, diferenciándolo del deporte en edad adulta.

Jiménez (2001), se pregunta ¿cuántos escolares están participando en los programas que ofrecen las distintas Administraciones?, ¿los que participan pertenecen a centros públicos o privados?, ¿practican más los niños o las niñas?, ¿en qué edades?, ¿qué tipo de actividad?...

Al observar la cantidad de niños y jóvenes que se interesan por el aprendizaje y práctica de algún deporte, mayoritariamente en torno al deporte escolar y próximo a su centro de enseńanza, nos da la sensación que es urgente y necesario conocer, estudiar y analizar lo que sucede en este ámbito.

A pesar de todo, son muy escasos los estudios realizados en relación al deporte escolar en la Región de Murcia, y más concretamente en relación a la participación. Cabe destacar el reciente estudio de López-Sánchez, González-Víllora \& Díaz-Suárez (2016) en el que se estudió el nivel de actividad física de nińos y adolescentes de la Región de Murcia, aunque en este estudio no se analizó la distinción entre centros públicos y concertados-privados. Por tanto, el presente estudio determinará cuáles son los niveles de participación de los escolares murcianos entre los cursos académicos 2005 al 2010, en relación al tipo de centro en el que están matriculados los alumnos, público o concertado-privado.

\section{Objetivo}

Diferenciar la participación del alumnado de centros privados-concertados en las competiciones del programa de Deporte Escolar, respecto a los de centros públicos.

\section{Metodología: Población y muestra}

La Población de estudio corresponde a todos los sujetos en Edad Escolar de la Región de Murcia, 741.728, siendo 379.336 (51,14\%) de género masculino y 362.392 (48,86\%) de género femenino, con una edad comprendida entre los 8 y los 18 años (Benjamín-Juvenil) durante los 5 cursos académicos objeto de esta investigación. Estas edades coinciden con 
las que se permite participar en el Campeonato de Promoción Deportiva.

La muestra está formada por 155.009 participaciones masculinas y 94.317 femeninas, todos ellos pertenecientes a centros docentes, tanto públicos como privados, y compitiendo en diferentes categorías (benjamín, alevín, infantil, cadete y juvenil). La muestra seleccionada corresponde a todas las participaciones que han realizado los sujetos en el Campeonato de Promoción Deportiva del programa de "Deporte Escolar" de la Región de Murcia, Un total de 249.326 participaciones de alumnos durante 5 temporadas (2005-2006, 2006-2007, 2007-2008, 2008-2009 y 2009-2010). La muestra se divide entre los 45 municipios de la Región de Murcia, incorporados a 8 grupos. Los sujetos en Edad Escolar están vinculados a un centro escolar o una entidad deportiva que se inscriba en este programa, no pudiendo estar ninguno de los sujetos que participan en el Campeonato de Promoción Deportiva inscritos en el Campeonato de Rendimiento de forma federada.. Es importante aclarar el concepto de ratio de participaciones por individuo de la población, se refiere a la media de participación por población, es decir, cuantas participaciones por individuo se producen en la población.

\section{Material e Instrumentos}

El estudio es fundamentalmente de tipo Descriptivo. Los datos se obtuvieron de la inscripción que se realiza de los alumnos desde el propio centro a través de la página web de Dirección General de Deportes de la Comunidad Autónoma de la Región de Murcia. En la herramienta "Gistur" diseńada para realizar la gestión de inscripciones vía online. Extrayendo de la misma la participación de los cursos académicos 2005-2006 hasta el 2009-2010. Una vez filtrada y limpiada la base de datos en Excel, después de realizar los diferentes filtros de actividades y codificar las variables a considerar, se volcaron al SPSS, versión 15.0 (IBM Corp. Released 2010. IBM SPSS Statistics for Windows, Version 15.0 Armonk, NY: IBM Corp.) para su posterior análisis. Maurandi, Del Río, y Balsalobre (2013), indican que es uno de los programas estadísticos más conocidos teniendo en cuenta su capacidad para trabajar con grandes bases de datos y un sencillo interface para la mayoría de los análisis. Además se empleó la hoja de cálculo CALC de la suite ofimática Libre Office.

Se construyeron tablas de frecuencias cruzadas y se analizaron las asociaciones mediante el test $\chi 2$ de Pearson y el test exacto de Fisher es una prueba de significación estadística utilizada en el análisis de tablas de contingencia. Aunque en la práctica se emplea cuando los tamaños de muestra son pequeños, también es válido para todos los tamaños de muestra.

Con los datos obtenidos en la investigación se han realizado las siguientes tablas y gráficas que nos aportan una idea clara de cuál ha sido la participación en el programa de Deporte Escolar en la Región de Murcia durante el período analizado 2005-2010.

\section{Resultados}

El número total de alumnos matriculados es de 193.628, en Centros Privados-Concertados de la Región de Murcia, alcanzando un $24,83 \%$ de participaciones en el programa de Deporte Escolar a lo largo de los cursos académicos objeto de este estudio. En relación a los Centros Públicos el número total de alumnos matriculados (548.100), realizando un $36,72 \%$ de participaciones en el programa de Deporte Escolar durante los cursos académicos objeto de este estudio.

En la Tabla 1, se expone la distribución de la muestra por tipo de centro y género, pudiendo apreciar que la participación es mayor en los centros públicos, en valores absolutos (201.242 frente a 48.084).

Tabla 1. Distribución de la muestra por Tipo de Centro y Género.

\begin{tabular}{|c|c|c|c|c|c|c|c|c|c|}
\hline & & & & \multicolumn{5}{|c|}{ Curso } & \multirow[b]{2}{*}{ Total } \\
\hline \multicolumn{3}{|c|}{ Tipo de Centro } & & $2005 / 06$ & $2006 / 07$ & $2007 / 08$ & $2008 / 09$ & $2009 / 10$ & \\
\hline \multirow[t]{6}{*}{ Participación } & Privado-Concertado & Género & Masculino & 4.432 & 5.999 & 6.184 & 7.440 & 7.203 & 31.258 \\
\hline & & & Femenino & 2.843 & 3.203 & 3.218 & 3.912 & 3.650 & 16.826 \\
\hline & & Total & & 7.275 & 9.202 & 9.402 & 11.352 & 10.853 & 48.084 \\
\hline & Público & Género & Masculino & 18.423 & 20.724 & 21.881 & 29.644 & 33.079 & 123.751 \\
\hline & & & Femenino & 12.165 & 13.879 & 13.660 & 18.330 & 19.457 & 77.491 \\
\hline & & Total & & 30.588 & 34.603 & 35.541 & 47.974 & 52.536 & 201.242 \\
\hline
\end{tabular}

El ratio de participaciones, Gráfica 1, demuestra que es mayor también en términos relativos 0,457 en los centros públicos frente a 0,268 en los centros privados-concertados. 
Gráfica 1. Ratio de participaciones por Tipo de Centro.

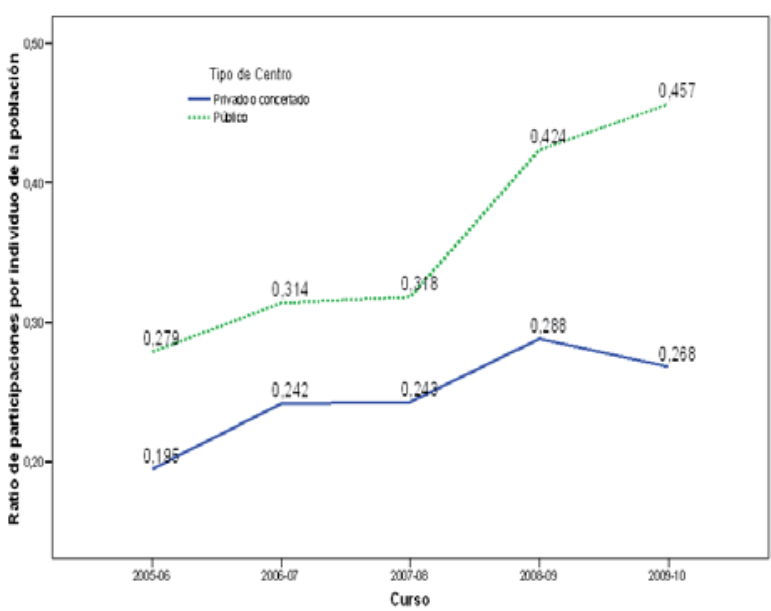

El patrón de crecimiento es similar entre los dos tipos de centro, salvo en el curso 2009/2010 que se observa un declive de participación en los centros privados, no dándose este en los centros públicos, desconociendo las causas de esta disminución en la participación, aunque esos años pertenecen a momentos de la profunda crisis económica sufrida en Espańa y como empresas que son los centros privados-concertados, puede entreverse que de una u otra manera también se vieron afectados.

\section{Discusión y conclusiones}

El objetivo de este estudio fue diferenciar la participación del alumnado de centros privados-concertados en las competiciones del programa de Deporte Escolar, respecto a los de centros públicos. Los resultados indican que nuestro estudio coincide con el Informe sobre el Deporte Escolar en Guipúzcoa (2010), donde la participación es mayor para los centros públicos, $48,5 \%$ frente al $42,3 \%$ de los privados, aunque esta diferencia es menor que en nuestra investigación. En cambio, el Observatorio del deporte de Sevilla (2007), obtiene en su estudio que los porcentajes de practicantes son muy similares en ambos tipos de centros, $70,72 \%$ en públicos y $72,86 \%$ en privados-concertados. Así como los resultados de Chinchilla et al. (2010), dónde la participación presenta las mayores diferencias ( $82,3 \%$ en centros públicos y $87,2 \%$ en concertados); similares a los de la "Fundació Jaume Bofill" (2009) (80,7\% en concertados y $75,6 \%$ en públicos).

La participación de los centros públicos frente a los privados-concertados en mayor tanto en términos relativos como en términos absolutos, en contra de lo que determina el INCE (2001), de forma general, los alumnos de centros concertados participan en actividades extraescolares en mayor proporción que los alumnos de los centros públicos.

Lo ideal sería poder conocer la participación de forma individual de cada alumno para que los datos fueran más certeros y poder así plantear otra serie de relaciones en cuanto a la participación.

Como limitaciones decir que la imposibilidad de conocer de forma individual la participación de cada alumno y en cada deporte, de forma clara, coarta la posibilidad de comparación con otros estudios de características similares, así como poder dimensionar la evolución de la participación a lo largo del tiempo y determinar si el modelo o programa de deporte en dad escolar da respuesta a las necesidades de los alumnos y por tanto cumple con la máxima de eficacia y eficiencia de un programa sostenido con fondos públicos.

\section{Referencias Bibliográficas}

1. Abad, M.T.; Robles, J. y Giménez, F.J. (2009). El deporte en las etapas educativas de primaria y secundaria. Revista Digital Lecturas: Educación Física y Deportes, 132. Mayo. Buenos Aires. Recuperado de http:// www.efdeportes.com/efd132/el-deporte-en-las-etapas-educativas. htm

2. Álamo, J.M. (2004). El deporte escolar en Canarias. En A. Fraile (Coord.), El deporte escolar en el siglo XXI: análisis y debate desde una perspectiva europea, (pp.133-152). Barcelona: Graó.

3. Blair, S., Dunn, A., García, M., Kampert,J., Kohl, H \& Marcus, B.(1999). Comparison of lifestyle and structured interventions to increase physical activity and cardiorespiratory fitness: a randomized trial. Jama. (27), 327-334.

4. Blázquez, D. (1995). La iniciación deportiva y el deporte escolar. Barcelona: INDE.

5. Bustamante, S. A., Chaverra, B., y González, E. V. (2011). La cultura corporal de nińos y niñas de una institución educativa. Saarbrücken, Alemania: Editorial Académica Española.

6. Cecchini, J. A., González, C., Méndez, A., Fernández-Río, J., Contreras, O. \& Romero, S. (2008). Metas sociales y de logro, persistencia-es- fuerzo e intenciones de práctica deportiva en el alumnado de Educación Física. Psicothema, 20(2), 260-265.

7. Devís, J. (1995). Deporte, educación y sociedad: hacia un deporte escolar diferente. Revista de Educación, 306, 455-472.

8. Díaz, A. y Martínez-Moreno, A. (2003). Deporte escolar y educativo. En Revista Digital Lecturas: Educación Fisica y Deportes, 67. Buenos Aires. Recuperado de http://www.efdeportes.com/efd67/educat.htm

9. Dirección General de Deportes de la Diputación foral de Guipúzcoa (2010). Informe sobre el deporte escolar en Guipúzcoa. Recuperado de http://www.kirolbegi.net/docs/reports/Informe_deporte_escolar_Gipuzkoa_09-10.pdf.

10. Elias, N., y Dunning, E. (1992). Deporte y ocio en el proceso de civilización. España: Fondo de Cultura Económica.

11. Fundació Jaume Bofill (2006). Debat familiar ¿Qué opinen les famílies sobre les activitats eductives fora de l'horari escolar? Barcelona: Ayuntament de Barcelona.

12. García-Aranda, J. (2010). Un deporte escolar para el futuro. En D. Blázquez (Coord.), La iniciación deportiva y el deporte escolar, (pp. 395-420). Barcelona: INDE. 
13. González, E. V., Padierna, J. C., Córdoba, M., Escobar, J. M., \& Gaviria, N. A. (2015). El deporte y la educación física escolar en Medellín Sport and school physical education in Medellín. SPORT TK: Revista Euroamericana de Ciencias Del Deporte, 55-69.

14. González, J. (2004). El deporte escolar en Cataluña. En A. Fraile (Coord.), El deporte escolar en el siglo XXI: análisis y debate desde una perspectiva europea, (pp.153-171). Barcelona: Graó.

15. Gutiérrez, M. (2000). Actividad física, estilos de vida y calidad de vida. Revista de Educación Física, 77, 5-14.

16. INCE (2001). Actividades del alumno fuera del horario escolar. Actividades extraescolares. Madrid: INCE. Recuperado de http://www.Ince. mec.es.

17. Jiménez, J.Ma. (2001). Deporte Escolar: Otros modelos. En Lillo, J. I.; Cotillas, F., y Pelayo, G. (Coord.), En Actas del I Congreso Deporte y Escuela, (pp. 11-36). Cuenca: Diputación Provincial de Cuenca.

18. Ley 2/2000, de 12 de Julio, del Deporte de la Región de Murcia. (2000). Revista Digital. Recuperado de http://www.galeon.com/deportedigital/ legislacion/ leymurc001.htm.

19. López, M. J. (2006). Características básicas del deporte en la escuela. Efdeportes, 11 (96), Recuperado de http://www.efdepor- tes.com/ efd96/escuela.htm.

20. López-Sánchez, G. F., González-Víllora, S. \& Díaz-Suárez, A. (2016). Level of habitual physical activity in children and adolescents from the Region of Murcia (Spain). SpringerPlus, 5:386.

21. Maurandi, A., Del Río, L. y Balsalobre, C. (2013). Fundamentos esta- dísticos para investigación. Introducción a R. Murcia. España. Bubok Publishing S.L. http://www.bubok.es/libros/223207/Fundamentosestadisticos-para-investigacionIntroduccion-a-R.

22. Moreno-Hernández, F.J. (1998). El deporte escolar como labor intencionada de intervención psicopedagógica y de aprendizaje. En VVAA. (COORD). Nuevos horizontes en la Educación Física y el Deporte Escolar. Málaga. Instituto Andaluz del Deporte.

23. Observatorio del Deporte de Sevilla. (2007). Hábitos y actitudes de los sevillanos en Edad Escolar ante el Deporte. Sevilla: Instituto Municipal de Deportes.

24. Pérez-Brunicardi, D. (2011). Buscando un modelo de deporte escolar para el municipio de Segovia. Un estudio a partir de las valoraciones, intereses $y$ actitudes de sus agentes implicados. Tesis doctoral. Valladolid: Universidad de Valladolid.

25. Piéron, M.; Telama, R.; Almond, L. y Carreiro da Costa, F. (1999). Estilo de vida de jóvenes europeos: Un estudio comparativo. Revista de Educación Física, 76, 5-13.

26. Rodríguez, J. J. (2010). La iniciación deportiva en el contexto escolar análisis de los modelos de enseńanza. Efdeportes, (142). Recuperado de http:/www.efdeportes.com/efd142/la-iniciacion-deportiva-en-elcontexto-escolar.htm

27. Sánchez, F. (1996). La actividad física orientada hacia la salud. Madrid. Biblioteca nueva.

28. Santos, M. y Sicilia, A. (1998). Actividades físicas extraescolares. Barcelona: INDE. 
